\title{
The Appliance of Duffing Chaotic Detection system in displacement measurement of capacitance sensor
}

\author{
Xiao Lin ${ }^{1, a}$, Ya-ping Dai ${ }^{2, b}$, Xing-xing $\mathrm{Hu}^{3, \mathrm{c}}$, Shu-gui $\mathrm{Wu}^{4, \mathrm{~d}}$ \\ ${ }^{1}$ School of Automation, Beijing Institute of Technology, Beijing, China \\ ${ }^{2}$ School of Automation, Beijing Institute of Technology, Beijing, China \\ ${ }^{3}$ Institute of Geophysics, China Earthquake Administration, Beijing, China \\ ${ }^{4}$ National Earthquake Infrastructure Service, China Earthquake Administration, Beijing, China \\ a601265041@qq.com, bdaiyaping@bit.edu.cn, chuxx05@sohu.com, dsgwu@neis.gov.cn
}

Keywords: duffing; chaos detection system; capacitance sensor; weak signal detection

\begin{abstract}
High precision micro displacement measurement framework is fundamental for drilling strain gauges. We try to combine Duffing Chaotic Detection (DCD) method to replace the traditional lock-in amplifier method in drilling strain gauges. Simulation results showed that the DCD method is better than lock-in amplifier method both in the detection accuracy and noise resistance. Even more, the design and realization of DCD method is easier than lock-in amplifier method.
\end{abstract}

\section{Introduction}

A drilling strain gauge is a precision scientific instrument which is used to measure the crust stress. It includes two orthogonal differential capacitance sensors. Capacitive sensors will translate the displacement caused by stress into electrical signal. And then stress can be calculated by processing related data[1]. The electrical signal is very weak, so we need to detect the $\mathrm{nV}$-level signals.

However, there are two questions in the actual measurement: (1) The amplitude of the signal is weak. (2) The signal is submerged by noise. These two questions lead to drilling strain gauges working under the condition of low measurement accuracy. The existed method to overcome this two questions is mainly the lock-in amplifier method[2]. The lock-in amplifier method is a kind of widely applied weak signal detection methods. It has nV-level measurement precision in theory, but the circuit is too complex and difficult to realize.

We try to combine Duffing Chaotic Detection (DCD) method to replace the traditional lock-in amplifier method in drilling strain gauges. DCD is one kind of applications of chaos in the weak signal detection. It can reach $\mathrm{nV}$-level precision and the circuit is simpler than the lock-in amplifier. The chaotic system is sensitive to sine signals of specific frequency. So it is used as a detection system face to weak signal[3,4]. Various parameters of the weak signal can be detected from the dynamic behavior of chaotic system through some appropriate signal processing methods[5].

In this article, we present a method--taking advantage of a DCD system to detect the weak signal from drilling strain gauges. Firstly, we introduce how differential capacitance sensor system translates the displacement to the electrical signal and the principle of the lock-in amplifier method. And then we discuss how to detect weak signals through the DCD method. On the basis of analyzing the composition of signal, we make simulation and compare the two methods.

\section{Differential Capacitance Sensor}

A differential capacitance sensor can translate a displacement to an electrical signal. The formula of capacitance is

$$
C=\frac{\varepsilon A}{d} \text {. }
$$


$\varepsilon$ is the dielectric constant. A is the area of the opposite plates. $\mathrm{d}$ is the spacing of plates.

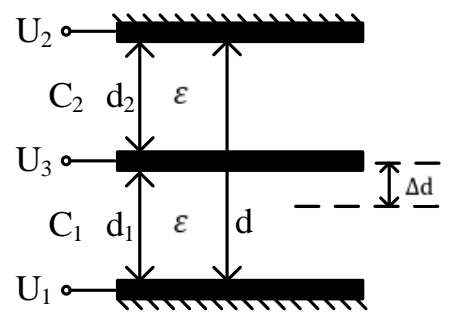

Fig.1 The differential capacitance

A differential capacitance is as shown in Fig.1. Two capacitances are $C_{1}$ and $C_{2}$. Two ends of the differential capacitance have the voltages of $U_{1}$ and $U_{2} . U_{3}$ is the signal produced in the middle plate. $\mathrm{d}_{1}$ and $\mathrm{d}_{2}$ are the spacing of the two capacitance respectively. When the $\mathrm{U}_{1}$ and $\mathrm{U}_{2}$ are sine signals with the same amplitude but opposite phase $\left(\mathrm{U}_{1}=-\mathrm{U}_{2}\right)$, by the thevenin's equivalent theorem and Eq.1, there is

$$
U_{3}=\frac{C_{1}}{C_{1}+C_{2}} U_{1}+\frac{C_{2}}{C_{1}+C_{2}} U_{2}=\frac{C_{1}-C_{2}}{C_{1}+C_{2}} U_{1}=\frac{d_{2}-d_{1}}{d_{2}+d_{1}} U_{1} .
$$

Suppose that the initial state of the differential capacitance is $C_{1}=C_{2}$. And at this time $d_{1}$ equals $\mathrm{d}_{2}$. We name the location of the middle plate as the zero position. $\Delta \mathrm{d}$ is the displacement of the middle plate away from the zero position. $d$ is the spacing of both ends and d equals the sum of $d_{1}$ and $\mathrm{d}_{2}$. We can suppose that $U_{1}=\mathrm{a} \sin \left(w_{0} t+\theta_{0}\right)$ and $U_{0}=\frac{2 a \Delta d}{d}$. Therefore $\mathrm{U}_{3}$ can be turned into

$$
U_{3}=\frac{d_{2}-d_{1}}{d} U_{1}=\frac{2 \Delta d}{d} U_{1}=\frac{2 a \Delta d}{d} \sin \left(w_{0} t+\theta_{0}\right)=U_{0} \sin \left(w_{0} t+\theta_{0}\right) .
$$

From Eq.3, we can see that if $\mathrm{d}$ and $\mathrm{U}_{1}$ are already known, $\mathrm{U}_{3}$ is proportional to the displacement of the middle plate $\Delta d$. The differential capacitance sensor is able to convert the displacement $\Delta d$ to the signal $U_{3}$. The frequency of $U_{3}$ equals the frequency of $U_{1}$ which can be produced by a oscillator. The amplitude of the signal $\mathrm{U}_{3}$ contains the location information $\Delta d$ of the middle plate.

\section{Lock-in Amplifier Method}

In a drilling strain gauge, the lock-in amplifier needs to detect the signal from the differential capacitance sensor. The block diagram of differential capacitance sensor system with lock-in amplifier method is shown in Fig.2. The input is the displacement of the middle plate $\Delta \mathrm{d}$. The output of the system is a DC signal $\mathrm{U}_{0}$, which is also the output of the lock-in amplifier.

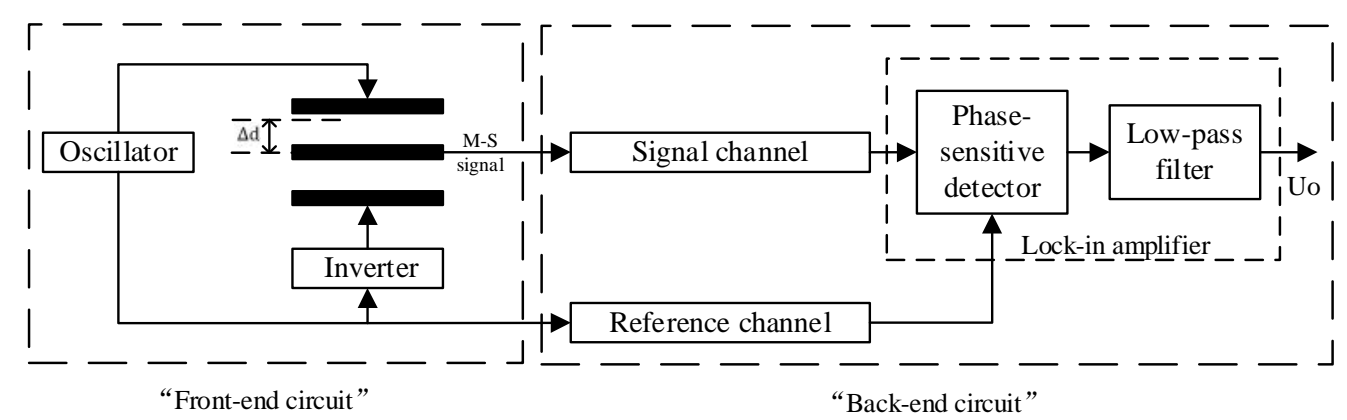

Fig.2 The block diagram of differential capacitance sensor system with lock-in amplifier method

The system is divided into two parts: the "front-end circuit" and the "back-end circuit"[6]. The "front-end circuit" includes the oscillator, differential capacitance and inverter, which is a differential capacitance sensor. The oscillator produces two ways of sine wave with fixed frequency. Assume one way is positive and another is reversed by an inverter. A same frequency Sine wave signal, naming it as M-S signal, will be produced in the middle plate of the capacitance. The "back-end circuit" 
includes signal channel, reference channel, phase-sensitive detector and low-pass filter. The M-S signal will be modulated and amplified by signal channel. Meanwhile the sine signal produced by oscillator is modulated by reference channel. The two ways of signal are input into the lock-in amplifier. A lock-in amplifier often consists of a phase-sensitive detector and a low-pass filter. But in practice, we generally use the orthogonal vector type lock-in amplifier[2] as shown in Fig.3, in order to make the demodulation process more rapid and more accurate.

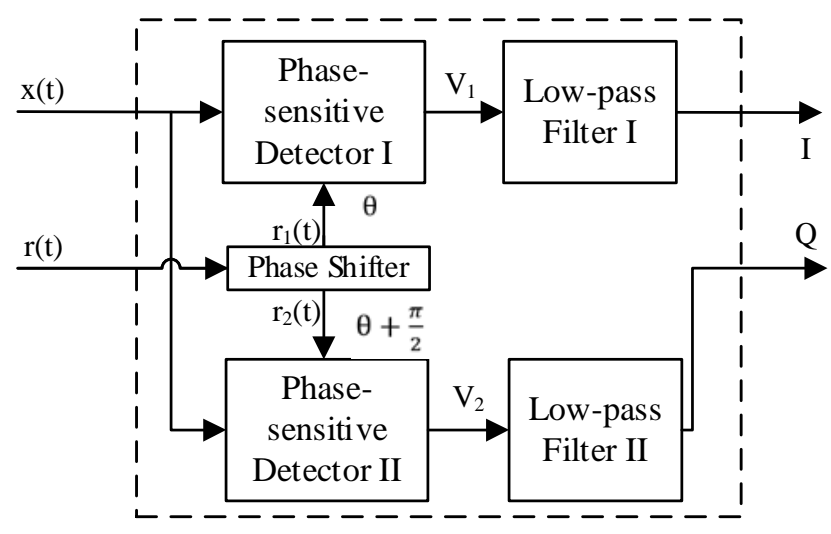

Fig.3 The block diagram of the orthogonal vector type lock-in amplifier

The inputs are the M-S signal and the reference signal. We name the M-S signal as $\mathrm{x}(\mathrm{t})$ and the reference signal as $\mathrm{r}(\mathrm{t})$. The M-S signal $\mathrm{x}(\mathrm{t})$ is input into Phase-sensitive Detector I and II respectively. The reference signal $\mathrm{r}(\mathrm{t})$ turns to two ways of sine wave with fixed frequency. Assume one way is $r(t)$ itself and the phase of another way is $\frac{\pi}{2}$ shifted by a phase shifter. The reference signal itself and another way are input into Phase-sensitive Detector I and II. We assume that the input signal is $\mathrm{x}(\mathrm{t})=U_{0} \sin \left(w_{0} t+\theta_{0}\right)$ and two reference signals are $r_{1}(t)=U_{r} \cos \left(\omega_{r} t+\theta_{r}\right)$ and $r_{2}(t)=U_{r} \sin \left(\omega_{r} t+\theta_{r}+\pi / 2\right)$. So the outputs of Phase-sensitive Detector I and II are $V_{1}=x(t) r_{1}(t)$ and $V_{2}=x(t) r_{2}(t)$. And then through Low-pass Filter I and II, we can peel out the DC components of $\mathrm{V}_{1}$ and $\mathrm{V}_{2}$, which are $\mathrm{I}=\frac{1}{2} U_{0} U_{R} \cos \left(\theta_{0}-\theta_{R}\right)$ and $\mathrm{Q}=\frac{1}{2} U_{0} U_{R} \sin \left(\theta_{0}-\theta_{R}\right)$ respectively. $\mathrm{I}$ and $\mathrm{Q}$ contain the amplitude information of M-S signal. Then we can calculate the sum of squares of I and Q through arithmetic circuit. We name it as $R=\sqrt{\left(I^{2}+Q^{2}\right)}=1 / 2 U_{0} U_{R}$. The amplitude of the reference signal $U_{\mathrm{R}}$ is already known. Because of $U_{0}=\frac{2 a \Delta d}{d}$, the displacement of the middle plate $\Delta$ d equals

$$
\Delta \mathrm{d}=\frac{U_{0} d}{2 a}=\frac{R d}{U_{r} a} .
$$

The lock-in amplifier method is simple and easy understood with high precision. But it also has many disadvantages: (1) the implementation of the following arithmetic circuit is very difficult; (2) a low-pass filter must keep certain bandwidth which may leave some residual noise; (3) the reference signal contains noise with the M-S signal's frequency and high order harmonic component. This leads to the detection threshold of lock-in amplifier cannot be further reduced. So researchers are trying to find a stable signal detection method to break through the minimum of detection threshold. DCD method has attracted more and more researchers' attention for its superiority.

\section{Duffing Chaotic Detection (DCD) Method}

Although the lock-in amplifier method is common used and has nV-level precision in theory, the circuit is too complex. What's more, the precision can't reach that high level in practice. So we introduce DCD method to replace lock-in amplifier method for drilling strain gauges. In this chapter, we firstly introduce the principle of DCD method; and then we introduce how to apply the DCD system to detect the M-S signal from the differential capacitance sensor. 


\section{The Principle of Duffing Chaotic Detection Method}

The Duffing chaotic detection (DCD) method is one kind of applications of chaos in the weak signal detection. Chaos' sensitivity to initial conditions and noise immunity make DCD method itself have $\mathrm{nV}$-level detection precision. It is based on Duffing chaotic equation. In order to detect the weak signal with any frequency under noise, The deformed Duffing chaotic equation is as follows

$$
\ddot{x}+\omega k \dot{x}-\omega^{2}\left(x-x^{3}\right)=a_{c} \cos \omega t+a_{x} \cos \omega t .
$$

$a_{c}$ is the amplitude of the drive signal. $a_{x}$ is the amplitude of the signal to be detected. In this paper, the signal to be detected is the M-S signal. $k$ is the damping ratio. $\omega$ is the frequency of the driving signal and M-S signal. According to Eq.5, we can establish the simulation model.

The drive signal and the M-S signal are the input of DCD system. The output of this system is the phase diagram. Suppose the M-S signal is zero and $\omega=10 \mathrm{rad} / \mathrm{s}$. When damping ratio $k$ is fixed (suppose $k=0.5$ ), the state of DCD system changes along with the driving signal regularly.

Firstly, set the driving signal $a_{c}$ to the chaos threshold $a_{d}$. When $a_{c}=a_{d}=0.825802654$, the system is in critical chaotic state, as shown in Fig.4. When $a_{c}=0.825802655$, the system turns into large scale periodic motion state, as shown in Fig.5. So the chaos threshold $a_{d}=0.825802654$. Set the driving signal $a_{c}$ to the chaos threshold $a_{d}$, making the DCD system stay in critical chaotic state.

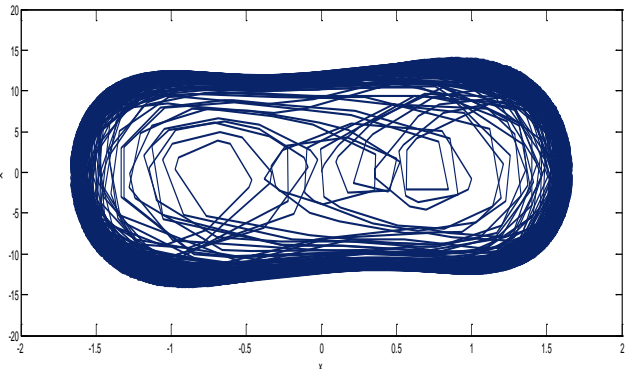

Fig.4 Critical state of chaos $\left(\omega=10 \mathrm{rad} / \mathrm{s}, a_{c}=0.825802654\right)$

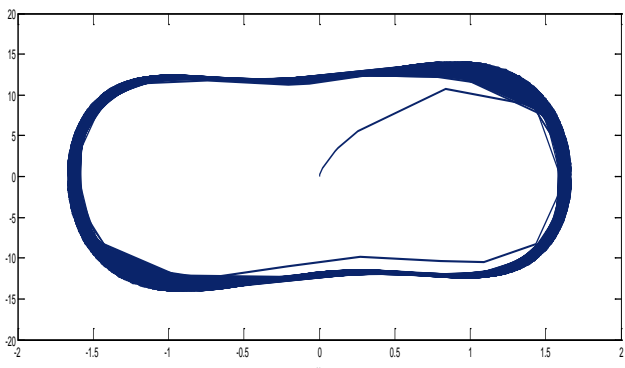

Fig.5 Large scale periodic motion state $\left(\omega=10 \mathrm{rad} / \mathrm{s}, a_{d}=0.825802654\right)$

Secondly, input the M-S signal into the DCD system. Suppose the amplitude of M-S signal is $a_{x}=0.000000001$. The state of system turns to large scale periodic motion state rapidly. If the signal doesn't contain the periodic signal with specific frequency and only contains noise, the system will remain in chaos.

Thirdly, decrease the drive signal's amplitude $a_{c}$, until the system turns back to chaos. Record the amplitude of the drive signal $a_{c}^{\prime}=0.825802653$. Then the amplitude of the M-S signal is $a_{x}=a_{c}-a_{c}^{\prime}=0.000000001$.

All in all, set the DCD system in critical chaotic state; take the M-S signal as a part of the driving signal to input into the DCD system; once there exists the periodic signal with specific frequency, even if it is very weak, the state of DCD system has a big change. By identifying system's phase diagram, we can judge whether the signal exists and obtain the amplitude.

\section{Application of Duffing Chaotic Detection System}

The voltage signal of differential capacitance sensor includes not only the M-S signal, but also a lot of noise. From Fig.2, we can see that the complex processes will introduce new noise and cause the loss of the M-S signal. In addition, the reference signal is not ideal sine wave which also includes noise. We need to try to simplify the circuit in order to detect the amplitude of weak signal with minimal steps.To solve these problems, we use the DCD system to replace the whole "back-end circuit”. The block diagram of DCD system for differential capacitance weak signal detection is shown in Fig.6: 


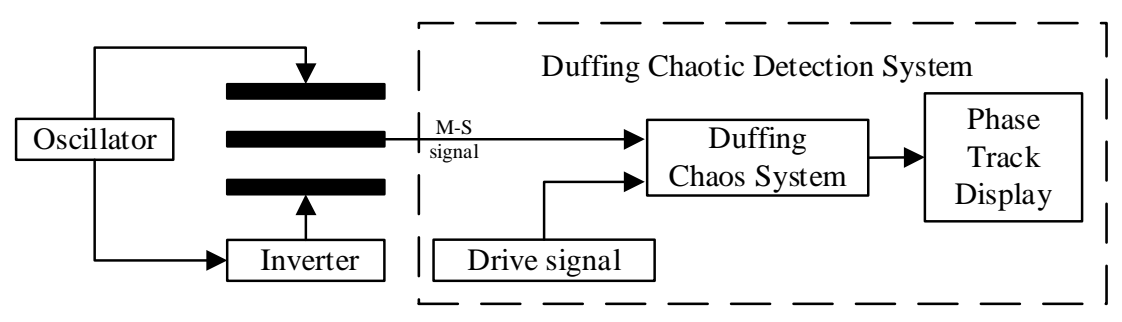

Fig.6 Duffing chaotic detection system for differential capacitance sensor

The design idea of the system is to detect weak signals directly or through some processes as few as possible. Compared with Fig.2, the middle plate produces the M-S signal, which connects to DCD system without amplification, filter and computing. By adjusting the amplitude of the driving signal and identifying the system state, the amplitude of the M-S signal can be obtained.

The system has the following advantages: (1) the amplitude of M-S signal can be obtained by DCD system with few signal processes. The weak signal can enter chaotic systems directly; (2) DCD system has a higher precision. This is determined by the initial value sensitivity of chaos. (3) DCD systems have a certain immunity to Gaussian noise and non-specific frequency signal.In the next chapter, we analysis the noise resistance ability of DCD system, and compare the DCD system and the lock-in amplifier method through simulation.

\section{Simulation and Analysis}

Suppose that the mixed signal $\mathrm{x}(\mathrm{t})=\mathrm{s}(\mathrm{t})+\mathrm{n}(\mathrm{t})+\mathrm{E}(\mathrm{t})+\mathrm{f}(\mathrm{t}): \mathrm{s}(\mathrm{t})$ : The $\mathrm{M}-\mathrm{S}$ signal $\mathrm{s}(\mathrm{t})=U_{0} \sin \left(\omega_{0} t\right)$. The frequency equals to the sine wave produced by oscillator; $\mathrm{n}(\mathrm{t})$ :Gaussian white noise: $\mathrm{E}(\mathrm{t})$ :The constant error caused by the amplitude difference between the differential capacitance sensor; $f(t)$ :Harmonic noise $f(t)=U_{2} \sin \left(2 \omega_{0} t\right)$. We only consider the affect of second harmonic noise.

According to the mathematical model and system block diagram, we establish the simulation model for the DCD system and the lock-in amplifier. Firstly, we analysis the noise resistance ability of DCD system. As for Gaussian noise, we make simulation for detection precision of two methods.

\section{Noise Resistance Ability of Duffing Chaotic Detection System}

Suppose that the amplitude of the input sine signal is $1 \mathrm{nV}$. The frequencies are $\omega_{0}=10 \mathrm{rad} / \mathrm{s}$ and $\omega_{0}=100 \mathrm{rad} / \mathrm{s}$. Input the constant error, harmonic noise and Gaussian white noise under different frequency condition respectively. We adjust the maximum strength of noise until DCD system can reach $n V$-level precision. Then we record it and compute the signal-noise ratio (SNR) at this moment. The SNR is the lower limit SNR of the input signal for $\mathrm{nV}$-level precision.

Table 1 The lower limit SNR of different frequency signals under different noise

\begin{tabular}{|c|c|c|c|c|}
\hline \multirow{2}{*}{$\begin{array}{c}\text { Signal } \\
\text { frequency } \\
{[\mathrm{rad} / \mathrm{s}]}\end{array}$} & $\begin{array}{c}\text { Signal amplitude } \\
{[\mathrm{nV}]}\end{array}$ & \multicolumn{3}{|c|}{ The lower limit SNR of the input signal under different noise [db] } \\
\cline { 3 - 5 } & 1 & Constant error & Second harmonics & Gaussian white noise \\
\hline 10 & 1 & -53 & -92 & -76 \\
\hline 100 & -7 & -46 & -53 \\
\hline
\end{tabular}

We can conclude that the noise resistance ability of DCD system to three types of noise is satisfactory. As the increase of the frequency, the lower limit SNR threshold also increase. But it can meet the measurement requirements in practice.

\section{Comparison of Duffing Chaotic Detection System and Lock-in Amplifier}

Gaussian white noise is a most common kind of noise. So we measured the lower limit SNR of DCD method and lock-in amplifier method for 1nV-level precision under Gaussian white noise. Suppose that the amplitude of the input sine signal is $1 \mathrm{nV}$. The frequencies are $\omega_{0}=10 \mathrm{rad} / \mathrm{s}$ and $\omega_{0}=100 \mathrm{rad} / \mathrm{s}$. Adjust the maximum strength of Gaussian white noise until DCD system and lock-in amplifier system can reach $1 \mathrm{nV}$-level precision. Then we record the data and compute the SNR of the input signal at this moment as shown in Table 2. 
Table 2 The lower limit SNR of DCD method and lock-in amplifier method for $1 \mathrm{nV}$-level precision

\begin{tabular}{|c|c|c|}
\hline \multirow{2}{*}{$\begin{array}{c}\text { Signal frequency } \\
{[\mathrm{rad} / \mathrm{s}]}\end{array}$} & \multicolumn{2}{|c|}{ The lower limit SNR of different method for 1nV-level precision [db] } \\
\cline { 2 - 3 } & DCD method & Lock-in amplifier method \\
\hline 10 & -76 & -30 \\
\hline 100 & -53 & -30 \\
\hline
\end{tabular}

From Table 2, we can see that when $\omega_{0}=10 \mathrm{rad} / \mathrm{s}$, SNR of DCD method has reach -76db while SNR of lock-in amplifier method is $-30 \mathrm{db}$. And when $\omega_{0}=100 \mathrm{rad} / \mathrm{s}$, SNR of DCD method has reach -53db while SNR of lock-in amplifier method is -30db. As the increase of signal frequency, the lower limit SNR of DCD method increases but is always lower than lock-in amplifier method. In other word, the detection precision of lock-in amplifier method is not as high as DCD method.

\section{Summary}

We have discussed the noise resistance of DCD method and compared two methods in the detection precision. Simulation results show that DCD method has strong ability to resist Gaussian white noise, the constant error and harmonic noise. Furthermore DCD method is more accurate than lock-in method in $1 \mathrm{nV}$ signal detection. When replacing the traditional lock-in amplifier method with DCD method in drilling strain gauges, the circuit is simplified at the same time. Obviously DCD method has a great application prospect.

In order to apply DCD method in weak signal detection for drilling strain gauges, we can further research in the following aspects: (1)Identify chaotic state of the system automatically. When we observe the phase diagram to identify system's state, manual errors cannot be avoided. So we can use a program to identify chaotic state of the system to avoid errors. (2)The pre-progressing of the M-S signal. Noise affects the system's precision seriously. We can seek a method that can pre-progress the M-S signal under weak signal condition to weaken some noise.(3)Implementation of DCD system circuit. We should complete the circuit implementation to test the method.

\section{References}

[1] Wen-ping Li, Lin-tao Li, Peng-fei Wu. Research on sinusoidal excitation source of capacitive micrometer for airborne gravity measurement system based on DDS technology[J]. Journal of Geodesy and Geodynamics, 2013, (S2):84-87.

[2] Jin-zhan Gao. Detection of weak signals[M]. Beijing: Tsinghua University Press, 2011.

[3] Chun-yan Nie. Chaotic Systems and Detection of Weak Signals[M]. Beijing: Tsinghua University Press, 2009.

[4] CHEN, Guan-rong. Chaos control and anti-control[J]. Guangxi: Journal Of Guangxi Normal University, 2002, 20(1):1-5.

[5] Yue Li, Bao-jun Yang. An Introduction to The Chaotic Detection [M]. Beijing: Publishing House Of Electronics Industry, 2004.

[6] Yan-zheng Bai, Ze-bing Zhou, Hai-bo Tu, Shu-chao Wu, Lin Cai, Li Liu, Jun Luo. Capacitive position measurement for high-precision space inertial sensor[J]. Frontiers of Physics in China, 2009, 4(2):205-208. 